\title{
Manifestações orais de arboviroses com ênfase em dengue, zika e chikungunya: revisão de literatura
}

Oral manifestations of arboviral diseases with emphasis on dengue fever, zika and chikungunya: literature review Manifestaciones orales de árboles con comienzo en dengue, zika y chikungunya: revisión de literatura Amanda Alves de OLIVEIRA ${ }^{1}$ Maria Gabriella de Abreu LACERDA ${ }^{\mathbf{1}}$

Ariana Alves de Oliveira BARROS ${ }^{1}$ Jaqueline Oliveira BARRETO

José Henrique de Araújo $\mathbf{C R U Z}^{1}$

Julliana Cariry Palhano FREIRE ${ }^{2}$

Eduardo Dias RIBEIRO ${ }^{3}$

${ }_{1}^{1}$ Departamento de Odontologia do Centro de Saúde e Tecnologia Rural, Universidade Federal de Campina Grande, UFCG, 58708-110 Patos - PB, Brasil ${ }^{2}$ Departamento de Odontologia, Universidade Estadual da Paraíba, UEPB, 58429-500 Campina Grande - PB, Brasil

${ }^{3}$ Departamento de Cirurgia e Traumatologia Bucomaxilofacial, Universidade Federal da Paraíba, UFPB, 58051-900, João Pessoa - PB, Brasil

\section{Resumo}

Introdução: A dengue, zika e chikungunya são doenças virais que podem ser classificadas como arboviroses. Suas complicações clínicas são de larga escala e podem acometer a cavidade oral. Objetivos: Realizar uma revisão na literatura científica sobre as manifestações clínicas na cavidade oral provocadas pelas arboviroses transmitidas pelo mosquito Aedes Egipty. Metodologia: foram analisados 69 artigos científicos obtidos nas bases de dados PubMed e Scielo correspondentes ao período de 2000 a 2018, tomando por base os seguintes descritores: "dengue", "zika" e "chikungunya" em associação com o termo "oral manifestation(s)". Resultados: Nas infecções por dengue clássica os relatos mais frequentes foram manifestações mucocutâneas, vermelhidão e edema nas mucosas, enquanto na dengue hemorrágica o sangramento gengival é tido como mais frequente seguido de focos hemorrágicos pelas demais regiões da mucosa bucal; já a zika mostrou-se presente na saliva, úlceras aftosas, paralisia facial e complicações orais relacionadas à microcefalia; nos casos de infecção pelo vírus chikingunya foram relatados dor, queimação e sangramento na gengiva, incapacidade de deglutir e mastigar, halitose e dor na abertura da boca. Conclusão: Há manifestações orais durante a infecção por Dengue, Zika e Chikungunya demostrando assim, a importância e a necessidade da realização de maiores pesquisas neste campo para instituição de protocolo clínico.

Descritores: Infecções por Arbovirus; Dengue; Zika Virus; Vírus Chikungunya; Manifestações Bucais.

\section{Abstract}

Introduction: The dengue Fever, Zika and Chikungunya are viral diseases which can be classified as arboviral diseases. Its clinical complications are large scale and can affect the oral cavity. Objectives: To carry out a review in the scientific literature on the clinical manifestations in the oral cavity caused by the arboviruses transmitted by the mosquito Aedes Egipty. Material and Methods: We analyzed 69 scientific articles obtained from the PubMed and Scielo databases for the period 2000 to 2018, based on the following descriptors: dengue, zika and chikungunya in association with the term "oral manifestation (s) ".Results: In infections caused by the classic dengue fever form, the most frequent reports were mucocutaneous manifestations, redness and swelling on the mucous membranes, and on the hemorrhagic dengue the gingival bleeding is considered the most frequent, followed by hemorrhagic foci at the rest of the regions in the oral mucous membranes; in infections originated from Zika disease were noticed the presence of the virus in the saliva, aphthous ulcers, facial palsy and oral complications related to microcephaly. And in cases of infections caused by Chikungunya virus, were reported pain, burning sensation and gingival bleeding, the inability of swallowing and chewing, halitosis and pain when opening the mouth. Conclusions: There are oral manifestations during the infection by Dengue, Zika and Chikungunya, thus demonstrating the importance and necessity of conducting further research in this field to establish a clinical protocol.

Descriptors: Arbovirus Infections; Dengue; Zika Virus; Chikungunya Virus; Oral Manifestations.

\section{Resumen}

Introducción: El dengue, zika y chikungunya son enfermedades virales que se pueden clasificar como arbovirosis. Sus complicaciones clínicas son de gran escala y pueden acometer la cavidad oral. Objetivos: Realizar una revisión en la literatura científica sobre las manifestaciones clínicas en la cavidad oral provocadas por las arbovirosis transmitidas por el mosquito Aedes Egipty. Metodologia: En el presente estudio se analizaron 69 artículos científicos obtenidos en las bases de datos PubMed y Scielo correspondientes al período de 2000 a 2018, tomando como base los siguientes descriptores: "dengue", "zika" y "chikungunya" en asociación con el término "oral manifestación (s) s)".Resultados: En las infecciones por dengue clásico los relatos más frecuentes fueron manifestaciones mucocultáneas, enrojecimiento y edema en las mucosas, mientras que en el dengue hemorrágico el sangrado gingival se considera más frecuente seguido de focos hemorrágicos por las demás regiones de la mucosa bucal; ya la zika se mostró presente en la saliva, úlceras aftosas, parálisis facial y complicaciones orales relacionadas a la microcefalia; en los casos de infección por el virus chikingunya se han notificado dolor, ardor y sangrado en las encías, incapacidad para deglutir y masticar, halitosis y dolor en la apertura de la boca.Conclusión: Hay manifestaciones orales durante la infección por Dengue, Zika y Chikungunya demostrando así la importancia y la necesidad de realizar mayores investigaciones en este campo para la institución de protocolo clínico.

Descriptores: Infecciones por Arbovirus; Dengue; Virus Zica; Virus Chikungunya; Manifestaciones Bucales.

INTRODUÇÃO

A dengue, zika e chikungunya são arboviroses transmitidas aos humanos por meio da picada do mesmo mosquito hematófago: fêmeas da espécie Aedes Aegypti. Uma vez que um dos vírus se instala no organismo humano é iniciada a infecção, expressando sintomas semelhantes como febre, cefaleia, artralgia, mialgia, erupções cutâneas e quadros hemorrágicos, que variam principalmente no que se refere à intensidade e eventual condição sistêmica do paciente, entretanto outras características clínicas e sintomatológicas são vista de forma específicas. As complicações clínicas ocasionadas pela infecção por arboviroses são de larga escala e podem acometer também a cavidade oral em boa porcentagem dos casos, gerando um desafio para a Odontologia ${ }^{1-4}$. 
A dengue é oriunda do vírus RNA da família Flaviviridae que possui diferentes tipos sorológicos, desde o leve ao mais severo, a saber DENV-1 a DENV-4. O vírus zika é um arbovírus da mesma família do vírus da dengue, do gênero Flavivirus, com duas linhagens descritas: africana e asiática e o Chikungunya (CHIKV) é um RNA vírus da família Togaviridae do gênero Alphavirus ${ }^{5}$. De acordo com Delatte et al. ${ }^{6}$, a infecção por flavivírus é mediada pela interação da partícula viral com o receptor celular após há endocitose e desnudamento, onde é feita a liberação do capsídeo e a liberação do RNA no citoplasma celular. Nos ribossomos é feita a tradução das proteínas virais e formação de novas fitas de RNA onde se encerra a replicação e após a montagem de novas partículas virais estas são liberadas da célula por exocitose.

No Brasil fatores como o clima e o crescimento populacional desordenado são favoráveis ao desenvolvimento e sobrevivência dos Aedes Aegypt, assim como ao surgimento de epidemias em determinadas épocas do ano. Dessa forma, se torna relevante as pesquisas que demonstram alterações no sistema estomatognático durante os frequentes surtos de dengue, zika e chikungunya ${ }^{7}$.

Dado o exposto, a presente revisão de literatura busca observar quais são as manifestações clínicas na cavidade oral provocadas pelas arboviroses transmitidas pelo mosquito Aedes Aegypt.

MATERIAL E MÉTODO

Realizou-se uma revisão da literatura no período de 20 de Janeiro a 15 de Março de 2019, onde foram analisados artigos científicos obtidos na base de dados PubMed (https://www.ncbi.nlm.nih.gov/pubmed) e Scielo (http://www.scielo.org), selecionando estudos publicados entre o período de 2000 a 2018, com os seguintes descritores: "Dengue", "zika" e "chikungunya" em associação com o termo "oral manifestation(s)". Após a leitura inicial dos títulos e resumos, 69 artigos se enquadraram dentro do objetivo do estudo. Posteriormente à leitura dos artigos completos, foram selecionados 43 artigos para embasamento desta revisão de literatura.

REVISÃO DA LITERATURA

\section{- Dengue}

Segundo Fernandes et al. $^{8}$ e Hasan et al. ${ }^{9}$, os principais sintomas específicos e não específicos apresentados na dengue são conhecidos, mas os sinais relacionados à cavidade oral não têm maiores evidências, principalmente associadas à dengue hemorrágica. Discordando com Joob e Wiwanitkit $^{10}$, o qual relata que tais manifestações são despercebidas pelo clínico geral, colaborando com Pedrosa et al. ${ }^{11}$ que destacou 0 envolvimento da mucosa oral durante o quadro de dengue como o achado mais frequente em suas pesquisas (66,67\%).

As manifestações orais observadas com prevalência na dengue clássica são alterações mucocultâneas: eritema e crostas em lábios e língua e pequenas vesículas no palato mole $^{12}$, além de lesões como hiperpigmentação ou vermelhidão da mucosa alveolar, edema e lesões maculopapulares no lábio inferior e na mucosa jugal ${ }^{13-14}$.

Em contrapartida, um estudo recente relatou que nos quadros de dengue hemorrágica as manifestações orais são de diferentes graus $^{15}$, sendo visto sangramento gengival relacionado ou não com a febre da dengue, possivelmente causado por trombocitopenia abaixo de $39.000 / \mathrm{mm}^{16}$. Nas regiões de língua e o palato mole também podem ser vistos eritema, petéquias $e$ equimoses $^{8}$, presença de placas hemorrágicas levantadas na mucosa bucal direita e esquerda, bem como no dorso da língua ${ }^{17}$ e numerosas bolhas hemorrágicas na mucosa sublingual, superfície lateral da língua, assim como casos de sangramento nasal ${ }^{18}$.

Outras manifestações relatadas desta arbovirose são: osteonecrose e reabsorção radicular, amígdalas ampliadas e inflamadas, xerostomia, sede excessiva e boca seca, sensação de gosto amargo, aumento da glândula parótida unilateralmente e sialodenite aguda e início súbito de disartria leve e dificuldade de engolir ${ }^{15,17,19-21}$. - Zika

Aspectos relevantes são notados para o vírus zika, principalmente no que se refere ao seu isolamento em fluídos corporais, como por exemplo, urina, sêmen, fluido crevicular e saliva $^{22}$, sendo esta última, uma forma de detecção com finalidade diagnóstica, auxiliando assim quando não há possibilidade de efetuar procedimentos de coleta mais invasivos como a de sangue ${ }^{23-24}$. Todavia, não existem indícios suficientes que comprovem sua transmissão por via salivar ${ }^{25}$.

Além de sua manifestação salivar a sintomatologia do ZIKV se diferencia das demais arboviroses pelo prolongamento das dores e edemas articulares e em uma pequena parcela dos acometidos pode acontecer complicações neurológicas como paralisia facial, surdez e Síndrome de Guillain-Barré, podendo causar também microcefalia ${ }^{26-32}$. 
Destacando a microcefalia, Broutet et al. ${ }^{33}$ relaciona-a diretamente com a infecção vertical por zika na gestação, e para bebês com esta condição, Varelli et al ${ }^{34}$ observaram alterações orais incluindo traumas dentários, doenças no periodonto, bruxismo, disfagia, atraso na erupção dentária e micrognatia. Além disso, duas crianças no nordeste do Brasil apresentaram fenda palatina e labial sem nenhum histórico familiar, isso fez com que aumentassem as suspeitas em relação a ação do $\mathrm{ZIKV}^{35}$.

Segundo Tappe et $a l .^{36}$ há surgimento de úlceras aftosas recorrente no meio oral, concordando com Foy et al. ${ }^{37}$ que observou isso na mucosa labial. Também se constatou hipoplasia de esmalte contraída por via sistêmica com lesões oscilates entre manchas discretas a um comprometimento significativo, ou lesões como manchas brancas, amareladas ou falhas no formato de depressões e fissuras ${ }^{38}$. - Chikungunya

$\mathrm{Na}$ cavidade oral foi comprovado a presença do vírus chikingunya (CHIKV) na saliva de macacos, camundongos e seres humanos. Contudo, diferencia-se do ZIKV por possuir indícios de transmissão pelo fluido salivar durante a fase aguda da doença segundo testes em camundongos, nos quais distúrbios hemorrágicos acometerem a cavidade nasal e oral, fazendo com que os glóbulos vermelhos drenassem para a boca ${ }^{25,39-44}$.

De modo geral, seus sinais e sintomas comumente relatados foram febre, cefaleia e dor nas articulações ${ }^{36}$, embora manifestações de menor escala também foram descritas: linfoadenopatia cervical ou generalizada, poliartrite crônica, manifestações neurológicas, encefalite e úlceras orais, náuseas, vômito, fotofobia, dor retro-orbital, conjuntivite e edema de face ${ }^{40-47}$

Embora os estudos estejam centrados na sintomatologia clássica ${ }^{48-51}$, na investigação de Katti et al. ${ }^{52}$, as manifestações orofaciais dos enfermos por chikungunya não são incomuns. Enfatizando em seu estudo que $54,32 \%$ dos pacientes diagnosticados com chikungunya apresentaram dor, sensação de queimação e sangramento gengival, $29,1 \%$ incapacidade de deglutir e mastigar, $21,34 \%$ halitose, $17,46 \%$ ulceração, incapacidade / dor na abertura da boca em $11,64 \%$, salivação excessiva em 9,7\% e descarga esbranquiçada das gengivas em $7,76 \%$.

RESULTADOS E DISCUSSÃO

Nos casos de infecções pelo vírus da dengue são mais recorrentes lesões mucocutâneas, hiperpigmentação, vermelhidão, edema avermelhado e lesões maculopapulares na cavidade oral ${ }^{11-14}$. Colaborando com Pincus et al. $^{53}$ que descreveu como principais sinais e sintomas encontrados na infecção por dengue clássica alterações na mucosa como eritemas e crostas. Já em pacientes cujo diagnóstico é de dengue hemorrágica Denis et al. $^{54}$ e Itoda et al. ${ }^{55}$ constataram que cerca de um décimo dos relatos de pacientes com dengue hemorrágica apresentam algum sinal na cavidade oral. Dentre as manifestações orais encontradas as mais frequentes são sangramento gengival, sangramento nasal e placas hemorrágicas orais $^{8-16}$. Acrescidos de achados como petéquias e bolhas hemorrágicas ${ }^{56}$.

$\mathrm{Na}$ literatura a virulência do ZIKV na saliva gera controvérsias ${ }^{57-59}$. Mas, quanto ao diagnóstico, o fluído salivar demostra-se promissor $^{60}$. E no que se refere à alterações do estado normal da cavidade bucal mais especificamente, o que se relata é a associação de úlceras aftosas ${ }^{36}$, concordando com Joob e Itoda et al. ${ }^{61}$. Além da sintomatologia clássica e manifestações bucais é relatado que pacientes com zika também podem desencadear Síndrome de Guillain-Barré e, quando transmitida de forma vertical, microcefalia ${ }^{30,32,33}$. Em consonância com alguns autores que associam o desenvolvimento destas condições com a febre da zika ${ }^{61-64}$.

Os casos acometidos por CHIV apresentaram como manifestações orais: gengivite, dor gengival, sensação de queimação, úlceras na mucosa oral, máculas hipopigmentadas, lesões em crosta nos lábios e ângulo da boca, pigmentação na mucosa, candidíase e incapacidade/dor na abertura da boca $^{49,54,64-66}$, principalmente em sua fase aguda, onde se desencadeia a chamada febre da chikugunya ${ }^{47,48}$. Nesta fase é descrito principalmente a presença de estados febris associados a dores articulares debilitantes ${ }^{68}$, colaborando com o estudo de Katti et al. ${ }^{52}$, o qual afirma que embora o envolvimento articular sinovial seja uma característica marcante da doença, as articulações temporomandibulares estão envolvidas em menos de $6 \%$ dos casos, confirmando com Rodriguez-Morales et al ${ }^{68}$ relata que há incapacidade ou dor na abertura da boca em cerca de $11,64 \%$ dos casos.

CONCLUSÃO

Com base nesta revisão da literatura comprovou-se a existência de alterações do estado normal da cavidade bucal durante a expressão do Flavivirus da dengue, zika e 
chikungunya, de forma que cada arbovirose apresenta sinais e sintomas particulares e grau de severidade variável, demonstrando a necessidade da realização de maiores pesquisas neste campo, uma vez que a observação da modificação de estruturas e compostos orais podem fornecer diagnóstico precoce e auxiliar na conclusão de casos complexos.

\section{REFERÊNCIAS}

1. Freitas ARR, Angerami RN, von Zuben APB, Donalisio MR. Introduction and transmission of Zika virus in Brazil: new challenges for the Americas. Rev Inst Med Trop Sao Paulo. 2016;58:24.

2. Joshi V, Mouyra D, Sharma Rc. Persistence of dengue-3 virus through transovarial transmission passage in successive generations of Aedes aegypti mosquitoes. Am J Trop Med Hyg. 2002;67(2):158-61.

3. Srikiatkhachorn A, Krautrachue A, Ratanaprakarn W et al. Natural History of Plasma Leakage in Dengue Hemorrhagic Fever. The Pediatric Infectious Disease Journal. 2007;26(4):283-90.

4. Leão JC, Marques C, Duarte Um, de Almeida OP, Porter S, Gueiros LA. Chikungunya fever: General and oral healthcare implications. Oral Disease. 2018;24(1-2):233-37.

5. Diawo D, Amadou A. Sall, Cheikh T. Diagne, Oumar F, Ousmane F, Yamar Ba, et al. Zika virus emergence in mosquitoes in southeastern Senegal, 2011.PlosOne. 2014;9(10):109442.

6. Delatte H, Paupy C, Dehecq JS, Thiria J, Failloux AB, Fontenille D. [Aedes albopictus, vetor de vírus chikungunya e dengue na ilha da reunião: biologia e controle]. Parasite. 2008; 15(1):3-13.

7. Kumar R, Sharma MK, Jain SK, Yadav SK, SinghalAK. Manifestações cutâneas da feb re Chikungunya : observações de um surto em um hospital de cuidados terciários no sudeste de Rajasthan , na Índia. Indian Dermatol Online J. 2017 8(5):336-42.

8. Fernandes $\mathrm{Cl}$, Perez LE, Perez DE. Uncommon oral manifestations of dengue viral infection. Braz J Otorhinolaryngol. 2020;86:S3-S5.

9. Hasan S, Jamdar SF, Alalowi M, Al Ageel Al Beaiji SM. Dengue virus: A global human threat: Review of literature. J Int Soc Prev Community Dent. 2016;6(1):1-6.

10. Joob B, Wiwanitkit V. Oral manifestations of dengue viral infection. Braz J Otorhinolaryngol. 2017;83(5):605.

11. Pedrosa M, Pierote JA, Lopes LD, Pompeu JG. Manifestações orais relacionadas à dengue. Rev Assoc Paul Cir Dent. 2017;71(1):21-4.

12. Chadwick D, Arch B, Wilder-Smith A, Paton N. Distinguishing dengue fever from other infections on the basis of simple clinical and laboratory features: Application of logistic regression analysis. J Clin Virol. 2006;35(2): 47-53.

13. Ávila MER, Rey YB, Valdés TA. Manifestaciones bucales del dengue. Acta Méd Centro. 2013;7(1):80-3.

14. Pontes FS, Frances LT, Carvalho $M$ V, Fonseca FP, Neto NC, do Nascimento LS, Pontes HA. Severe oral manifestation of dengue viral infection: a rare clinical description. Quintessence Int. 2014;45(2): 151-56.

15. Al-Namnam NM, Nambiar $P$, Shanmuhasuntharam P, Harris M. A case of dengue-related osteonecrosis of the maxillary dentoalveolar bone. Aust Dent J. 2017;62(2): 228-32.

16. Joob B, Wiwanitkit V. Dengue and acute gingival bleeding. J Indian Soc Periodontol. 2014;18(1):4.

17. Mitra R, Baskaran $P$, Sathyakumar $M$. Oral presentation in hemorrhagic dengue: a rare entity. J Nat Sci Biol Med. 2013;4(1):264-67.

18. Amit Byatnal, Neha Mahajan, Shrinivas Koppal, Um Ravikiran, Thriveni R, Parvathi Devi MK. Unusual yet isolated oral manifestations of persistent thrombocytopenia: a rare case report. Braz J Oral Sci. 2013; 12(3):233-36.

19. Mattos NF, Santos NA, Wingert GC, Pies S, Lara GM, Carvalho TS. Dengue: Uma visão imunológica. Newslab. 2015; 5(8):3-4.

20.Denis CK, Cavalcanti KM, Meirelles RC, Martinelli B, Valença DC. Otolaryngological manifestations of patients with dengue. Bras Otorrinolaringol.2019;69(5):644-47.

21.Jaganathan $S$, Raman $R$. Hypoglossal nerve palsy: A rare consequence of dengue fever. Neurol Índia.2014;62(5):567-68.

22. Musso D, Teissier A, Rouault E, Teururai $S$, de Pina JJ, Nhan TX. Detection of chikungunya virus in saliva and urine. Virol J. 2016;13:102.

23. Nicastri E, Castilletti C, Liuzzi G, lannetta M, Capobianchi MR, Ippolito G. Persistent detection of Zika virus RNA in semen for six months after symptom onset in a traveller returning from Haiti to Italy, February 2016. Euro Surveill. 2016;21(32):303-14.

24. Fantinato $F$, Fontana $S T$, Lima Araújo L, Ribeiro IG, Andrade MR, Dantas ALM, et al.Descrição dos primeiros casos de febre pelo vírus Zika investigados em municípios da região Nordeste do Brasil, 2015. Epidemiol Serv Saúde. 2016;25(4): 683-90.

25. Bonaldo MC, Ribeiro IP, Lima NS, Santos AAC, Menezes LSR, Cruz SOD et al. Isolation of Infective Zika Virus from Urine and Saliva of Patients in Brazil. Plos Negl Trop Dis. 2016;10(6):4816. 
26. Leão JC, Gueiros LA, Lodi G, Robinson NA, Scully C. Zika virus: oral healthcare implications. Oral Dis. 2017;23(1):12-7.

27. Smith DW, Mackenzie J. Zika virus and Guillain-Barré syndrome: another viral cause to add to the list. Lancet. 2016;387(10027): 1486-88.

28. Cao-Lormeau V, Blake A, Mons $S$, Lastère $S$, Roche C, Vanhomwegen J et al. Guillain-Barré Syndrome outbreak associated with Zika virus infection in French Polynesia: a case-control study. Lancet. 2016; 387(10027) 1531-39.

29. Schuler-Faccini L, Ribeiro EM, Feitosa IML, Cavalcaante DP, Pessoa A, Doriqui MJR, et al. Possible Association Between Zika Virus Infection and Microcephaly - Brazil, 2015. MMWR Morb Mortal Wkly Rep. 2016;65(3): 59-62.

30.Kohashi BSO, Ribeiro EOA, Soares KS, Prestes GBR. Abordagem preventiva e educativa em paciente odontológico com microcefalia associada ao Zika virus: relato de caso. Arch Health Invest. 2019;8(1):33-8

31. Queiroz VKP, Soares DM. Pesquisas odontológicas relacionadas com microcefalia: um estudo bibliométrico. Arch Health Invest. 2019;8(11):681-85.

32. Freitas PSS, Bussinger ECA, Lacerda LCX, Soares GB, Maciel ELN. O surto de Zika vírus: produção científica após Declaração de Emergência Nacional em Saúde Pública. Arch Health Invest. 2018;7(1):12-6.

33. Broutet N, Krauer F, Riesen M, Khalakdina A, Almiron M, Aldighieri S, Espinal M, Low N, Dye C. Zika Virus as a Cause of Neurologic Disorders. N Engl J Med. 2016;374(16):1506-9.

34. Leite CN, Varellis MLZ. Microcefalia e a odontologia brasileira. J Health NPEPS. 2016; 1(2):297-304.

35. Moura da Silva AA, Ganz JS, Sousa $\mathrm{PD}$, Doriqui MJ, Ribeiro MR, Branco MD. Early Growth and neurologic outcomes of Infants with probable congenital Zika Virus Syndrome. Emerg Infect Dis. 2016; 22(11):1953-56.

36. Tappe D, Nachtigall S, Kapaun Um, Schnitzler P, Günther S, Schmidt-Chanasit J. Acute Zika virus infection after travel to Malaysian Borneo, September 2014. Emerg Infect Dis. 2015; 21(5):911-13.

37. Foy BD, Kobylinski KC, Foy JL, Blitvich BJ, Travassos da Rosa $A$, Haddow $A D$, et al. Provável Transmissão Não Vetorial de Zika Virus. Emerg Infect Dis. 2011;17(5):880-82.

38. Consolaro A, Francischone LA, Consolaro RB. Hipoplasia do esmalte: fundamentos para nomenclatura e identificação dos tipos e causas. Revista Dental Press de Estética. 2011;8(3):126-34.

39. Gardner J, Rudd PA, Prow NA, Belarbi E, Roques $\mathrm{P}$, Larcher $\mathrm{T}$ et al. Infectious chikungunya virus in the saliva of mice, monkeys and humans. PLoS One. 2015;10(10):e0139481.

40. Powers AM, Logue $\mathrm{CH}$. Changing patterns of chikungunya virus: re-emergence of a zoonotic arbovirus. J Gen Virol. 2007;88(Pt 9):2363-77.

41. Thiberville SD, Moyen N, Dupuis-Maguiraga L, Nougairede A, Gould EA, Roques $P$, de Lamballerie $X$. Chikungunya fever: epidemiology, clinical syndrome, pathogenesis and therapy. Antiviral Res. 2013;99(3):345-70.

42. Chopra A, Anuradha V, Ghorpade R, Saluja M. Acute Chikungunya and persistent musculoskeletal pain following the 2006 Indian epidemic: a 2-year prospective rural community study. Epidemiol Infect. 2012;140(5):842-50.

43. Chopra A, Anuradha V, Lagoo-Joshi V, Kunjir V, Salvi S, Saluja M. Chikungunya virus aches and pains: an emerging challenge. Arthritis Rheum. 2008;58(9):2921-2.

44. Madariaga $M$, Ticona $E$, Resurrecion C. Chikungunya: bending over the Americas and the rest of the world. Braz J Infect Dis. 2016;20(1):91-8.

45. Manimunda SP, Vijayachari $P$, Uppoor $R$, Sugunan AP, Singh SS, Rai SK et al. Clinical progression of chikungunya fever during acute and chronic arthritic stages and the changes in joint morphology as revealed by imaging. Trans R Soc Trop Med Hyg. 2010;104(6):392-99.

46. Simon F, Javelle E, Cabie A, Bouquillard E, Troisgros $\mathrm{O}$, Gentile $\mathrm{G}$ et al. French guidelines for the management of chikungunya (acute and persistent presentations). November 2014. Med Mal Infect. 2015;45(7):243-63.

47. Suryawanshi SD, Dube $A H$, Khadse RK, Jalgaonkar SV, Sathe PS, Zawar SD et al. Clinical profile of chikungunya fever in patients in a tertiary care centre in Maharashtra, India. Indian J Med Res. 2009;129(4):438-41.

48. Lahariya C, Pradhan SK. Emergence of the Chikungunya virus in the Indian subcontinent after 32 years: a review. J Vect Borne Dis. 2006; 43:151-60.

49. Mourya D, Yadav P. Vector biology of dengue \& chikungunya viruses. Indian J Med Res. 2006; 124(5):475-80.

50. Agarwal A, Joshi G, Nagar DP, Sharma AK, Sukumaran D, Pant SC et al. Mosquito saliva induced cutaneous events augment Chikungunya virus replication and disease progression. Infect Genet Evol. 2016;40:12635.

51. Lum FM, Ng LF. Cellular and molecular mechanisms of chikungunya pathogenesis. Antiviral Res. 2015;120:165-74.

52. Katti R, Shahapur PR, Udapudi KL. Impact of chikungunya virus infection on oral health status: An observational study.Indian J Dent Res. 2011;22(4):613. 
53. Pincus LB, Grossman ME, Fox LP. The exanthem of dengue fever: Clinical features of two US tourists traveling abroad. J Am Acad Dermatol. 2008;58(2):308-16.

54. Denis CK, Cavalcanti KM, Meirelles RC, Martinelli B. Manifestações otorrinolaringológicas em pacientes com dengue. Rev Bras Otorrinolaringol. 2003;69(5): 644-47.

55. Itoda I, Masuda G, Suganuma A, Imamura A, Ajisawa A, Yamada K, Yabe S, Takasaki T, Kurane I, Totsuka K, Negishi M. Clinical features of 62 imported cases of dengue fever in Japan. Am J Trop Med Hyg. 2006 Sep;75(3):470-74.

56. Lolekha R, Chokephalbulkit K K, Yoksan S, Vanprapar N, Phongsmart W, Chearskul S.Diagnosis of dengue infection using various diagnostic tests in the early stage of illness. J Trop Med Public Health. 2004;35(2):391-95.

57. Siqueira WL, Moffa EB, Mussi MC, Machado MA. Zika virus infection spread through salivaa truth or myth? Braz oral res. 2016;30(1):46

58. Hayes, Edward B. Zika Virus Outside Africa. Emerging Infectious. Diseases. 2015; 15(9):1347-50.

59. Musso D, Teissier A, Rouault E, Teururai S, de Pina JJ, Nhan TX. Detection of Zika virus in saliva and urine Virol J. 2016;13(1):120.

60. Bingham AM, Cone M, Mock V, HeberleinLarson L, Stanek D, Blackmore C, et al.Comparison of test results for Zika Virus RNA in urine, serum, and saliva specimens from persons with travel-associated Zika Virus Disease - Florida, 2016. MMWR Morb Mortal Wkly Rep.2016;65(18):475-78.

61. Itoda I, Masuda G, Suganuma A, Imamura A, Ajisawa $A$, Yamada $K$ et al. Clinical features of 62 imported cases of dengue fever in Japan. Am J Trop Med Hyg. 2006;75(3): 470-74.

62. Hamel $R$, Liégeois $F$, Wichit $S$, Pompon $J$, Diop $F$, Talignani $L$ et al. Zika virus: epidemiology, clinical features and host-virus interactions. Microbes Infect. 2016;18 (78):441-49.

63. Oehler E, Watrin L, Larre P, Leparc-Goffart I, Lastere S, Valour F, Baudouin L, Mallet $\mathrm{H}$, Musso D, Ghawche F. Zika virus infection complicated by Guillain-Barre syndrome--case report, French Polynesia, December 2013. Euro Surveill. 2014;19(9):20720.

64. Mysorekar IU, Diamond MS. Modeling Zika Virus Infection in Pregnancy. N Engl J Med. 2016;375(5):481-84.

65. Bandyopadhyay D, Ghosh SK. Mucocutaneous manifestations of Chikungunya fever. Indian J Dermatol. 2010;55(1):64-7.

66. Coffey LL, Failloux AB, Weaver SC. Chikungunya virus-vector interactions. Viruses. 2014;6(11):4628-63.
67. Couderc T, Lecuit M. Chikungunya Virus pathogenesis: From bedside to bench. Antiviral Res. 2015;121:120-31.

68. Rodríguez-Morales AJ, Cardona-Ospina JA, Fernanda Urbano-Garzón S, Sebastian Hurtado-Zapata J. Prevalence of PostChikungunya Infection Chronic Inflammatory Arthritis: A Systematic Review and MetaAnalysis. Arthritis Care Res (Hoboken). 2016;68(12):1849-58.

\section{CONFLITO DE INTERESSES}

Os autores declaram não haver conflitos de interesse

\section{AUTOR PARA CORRESPONDÊNCIA}

\author{
Amanda Alves de Oliveira \\ Departamento de Odontologia \\ Universidade Federal de Campina Grande \\ Avenida Universitária, S/N, 58708-110 \\ Patos, PB, Brazil. \\ Tel: +55-84-86295233 \\ E-mail: amandaoliveiraalves3@gmail.com
}

Submetido em 04/05/2019 Aceito em 23/10/2020 